Artigo Original

\section{Original Article}

\author{
Cássia Sígolo ${ }^{1}$ \\ Cristina Broglia Feitosa de Lacerda²
}

\title{
Da suspeita à intervenção em surdez: caracterização deste processo na região de Campinas/SP
}

\author{
From suspicion to intervention in deafness: characterization \\ of this process in Campinas/SP
}

\author{
Descritores \\ Perda auditiva \\ Diagnóstico precoce \\ Criança \\ Saúde da criança
}

Keywords

Auditory loss

Early diagnosis

Child

Child health
Endereço para correspondência: Cássia Sígolo

R. 24 de março, 273, Jardim Serrano, Serra Negra (SP) Brasil, CEP: 13930-000.

E-mail: csigolo@gmail.com

Recebido em: 22/9/2009

Aceito em: 18/8/2010

\section{RESUMO}

Objetivo: Identificar e detalhar a situação do diagnóstico e atendimento de crianças surdas de dois municípios que pertencem à região administrativa de Campinas (SP). Métodos: Foi realizado levantamento de 320 prontuários referentes à pacientes com diagnóstico de surdez pré-lingual no período de 1996 a 2005, em duas instituições localizadas na região de Campinas: uma clínica especializada no atendimento à surdez e uma clínica universitária. Resultados: Quanto a suspeita da perda auditiva, a média de idade foi de um 1 e 9 meses. A média de idade da primeira consulta com o médico ou fonoaudiológico foi de 3 anos e 6 meses; para o diagnóstico da surdez 4 anos e 3 meses; para o início de intervenção clínica 6 anos e 1 mês; e para a adaptação de aparelho de amplificação sonora individual (AASI) 7 anos e 5 meses. Conclusão: A suspeita, primeira consulta médica, diagnóstico, intervenção e adaptação de AASI ocorreram tardiamente, se considerados os padrões diagnósticos e de acesso aos serviços preconizados na atualidade. Além disso, há um intervalo de tempo importante entre cada uma das etapas, destacando-se principalmente o período entre a suspeita da surdez e o início da intervenção clínica. A região de Campinas é bastante desenvolvida economicamente, dispõe de uma ampla rede de serviços de saúde, mas se mostra pouco eficiente no que se refere ao atendimento em surdez.

\begin{abstract}
Purpose: To identify and detail the current situation of diagnosis and assistance to deaf children in two cities belonging to the administrative region of Campinas (SP). Methods: It was conducted a survey of 320 medical records of patients diagnosed with prelingual deafness in the period between 1996 and 2005, in two institutions located in the region of Campinas: a university clinic and a clinic specialized in the assistance to deaf individuals. Results: Regarding the suspicion of hearing loss, the average age of the subjects was 1 year and 9 months. The mean age of children referred to medical or speech-language pathology service was 3 years and 6 months; the mean age for deafness diagnosis was 4 years and 3 months; the mean age for the beginning of clinical intervention was 6 years and 1 month; and the mean age for hearing aid adaptation was 7 years and 5 months. Conclusion: Suspicion of deafness, the first visit to a doctor, diagnosis, intervention and hearing aid adaptation were all delayed when compared to current recommended diagnostic standards and conditions of access to services. In addition, there was a significant delay between each stage, especially in the period between the suspicion of deafness and the beginning of clinical intervention. Although Campinas region is well developed in economic terms, offering a broad network of health assistance services, this network was little efficient regarding assistance to deafness.
\end{abstract}

Trabalho realizado no Programa de Pós-graduação (Mestrado) em Saúde da Criança e do Adolescente, Universidade Estadual de Campinas - UNICAMP - Campinas (SP), Brasil, com bolsa concedida pela Coordenação de Aperfeiçoamento de Pessoal de Nível Superior (CAPES).

(1) Programa de Pós-graduação (Mestrado) em Saúde da Criança e do Adolescente, Universidade Estadual de Campinas - UNICAMP - Campinas (SP), Brasil.

(2) Pós-graduação (Doutorado) em Educação, Universidade Estadual de Campinas - UNICAMP - Campinas (SP), Brasil. 


\section{INTRODUÇÃO}

A audição é um dos sentidos que traz informações importantes para o desenvolvimento humano. Portanto, são várias as implicações decorrentes de uma perda auditiva, principalmente no que diz respeito ao desenvolvimento da linguagem e da comunicação oral.

Quanto mais cedo o diagnóstico da surdez, maiores as possibilidades de intervenção e auxílio no desenvolvimento sociocognitivo do sujeito. A audição é essencial à organização do pensamento e à compreensão do mundo e do outro, e faz parte de uma das funções superiores mais nobres do ser humano, que é a comunicação ${ }^{(1,2)}$.

Estudos realizados em 1998 e 1999 apontam que crianças que foram diagnosticadas até os seis meses de idade e logo em seguida (dois meses) iniciaram processo de intervenção, apresentaram desempenho de linguagem expressiva e receptiva dentro do esperado, quando avaliadas aos 26 meses. As crianças diagnosticadas após seis meses, com intervalo de intervenção consequentemente maior, apresentaram atraso significativo (de 12 a 14 meses) na linguagem expressiva e receptiva, quando comparadas às ouvintes ${ }^{(3-5)}$.

A literatura também aponta a relevância da intervenção fonoaudiológica antes dos seis meses de vida para melhor prognóstico quanto ao desenvolvimento de linguagem. Crianças, cujo diagnóstico das alterações auditivas foram realizados no primeiro semestre de vida, apresentam desenvolvimento cognitivo, de fala e de linguagem superior aos de crianças diagnosticadas após esse período ${ }^{(6)}$.

O sistema nervoso central apresenta grande plasticidade quando precocemente estimulado, principalmente, até os seis meses de idade, levando ao aumento das conexões nervosas e, consequentemente, melhor reabilitação das vias auditivas ${ }^{(7)}$. Portanto, até os seis meses de idade, o sistema auditivo central pode sofrer modificações positivas ou negativas, dependendo da quantidade e qualidade dos estímulos apresentados e capta$\operatorname{dos}^{(8)}$. A detecção precoce da surdez torna-se então imprescindível, uma vez que aumenta a probabilidade de se aproveitar ao máximo o potencial para a linguagem expressiva e receptiva, para o desempenho acadêmico e para o desenvolvimento social e emocional das crianças ${ }^{(9,10)}$.

Em virtude da importância do diagnóstico precoce de surdez e do conhecimento do desenvolvimento da linguagem em crianças surdas, faz-se necessário analisar a realidade quanto à identificação e ao diagnóstico precoce. Assim, será possível criar e realizar ações que previnam o atraso no desenvolvimento da linguagem de crianças surdas.

Este trabalho teve como objetivo identificar e detalhar a situação do diagnóstico e atendimento médico e fonoaudiológico a crianças surdas de dois municípios pertencentes à Região Administrativa de Campinas (SP).

\section{MÉTODOS}

O presente estudo foi aprovado pelo Comitê de Ética em Pesquisa da Faculdade de Ciências Médicas da Universidade Estadual de Campinas, sob protocolo 600/04.Trata-se de pes- quisa retrospectiva, realizada em duas instituições: uma clínica especializada no atendimento à surdez localizada na cidade de Campinas, e uma clínica-escola universitária localizada na cidade de Piracicaba. Ambas as instituições fazem parte da Região Administrativa e Metropolitana de Campinas ${ }^{(11)}$. Além disso, são referências de atendimento na região, atendem gratuitamente ou cobram valores segundo a renda mensal familiar e, por isso, tornam o atendimento acessível a todas as classes sociais. Todavia, a maior parte da população que procura por estes serviços é de baixa renda.

Para a coleta de dados foram utilizados os prontuários de pacientes atendidos entre 1996 e 2005, com diagnóstico de surdez pré-lingual. Na universidade, foram coletadas informações de 114 prontuários de pacientes atendidos no período eleito para a investigação. A clínica universitária não é equipada para o serviço de diagnóstico completo da surdez (não possui emissões otoacústicas evocadas - EOAE e potencial evocado auditivo de tronco encefálico - PEATE) e quando estas avaliações se fazem necessárias os pacientes são encaminhados para outros serviços, em geral, localizados na cidade de Campinas que conta com hospitais de grande porte e serviços especializados. $\mathrm{O}$ foco do serviço universitário é o atendimento clínico (fonoterapia) para os sujeitos, além de dispor também de serviço de indicação, seleção e adaptação de AASI. No entanto, mas não fornece as próteses auditivas, encaminhando os pacientes para serviços que o fazem.

Da clínica especializada no atendimento à surdez foram utilizados para o estudo 206 prontuários. Esta foi fundada na cidade de Campinas no final da década de 1980 com o objetivo de atender as necessidades de famílias que enfrentavam a falta de recursos para o atendimento do sujeito surdo. Atualmente, a clínica especializada funciona em prédio próprio, possui infraestrutura de salas, consultórios, e conta com atendimento nas áreas de Serviço Social, Audiologia Clínica, Otorrinolaringologia, Audiologia Educacional (Fonoterapia) e Pedagogia e é considerada um centro de referência em Campinas e região na área da surdez. Atende pessoas de todas as faixas etárias. O perfil dos usuários é variado e vem aumentando a procura de crianças e adolescentes por atendimento fonoaudiológico. Além disso, no período da coleta de dados do presente trabalho, era realizado o serviço de seleção, indicação, adaptação e fornecimento de AASI providos pelo Sistema Único de Saúde (SUS) em parceria com os serviços de duas universidades do município.

Neste contexto, a partir do material coletado na clínica especializada e na clínica universitária, foram considerados 320 prontuários. Os prontuários dos pacientes de ambos os serviços eram divididos em função do caráter multidisciplinar da equipe, e foram analisados os seguintes itens: ficha de cadastro; serviço social; anamnese fonoaudiológica; avaliação otorrinolaringológica; avaliação fonoaudiológica envolvendo indicação e/ou adaptação de AASI; relatório de evolução clínica de fonoterapia. Foi utilizado um protocolo para a coleta dos dados (Anexo 1).

Foram estudadas as seguintes informações: gênero; profissão e escolaridade dos pais; cidade de procedência; idade em que a surdez foi suspeitada; como foi a suspeita da surdez; quem suspeitou; idade em que foi pela primeira vez ao médico ou fonoaudiólogo; especialidade deste profissional; tipo e grau de perda auditiva encontrada, por orelha; etiologia da surdez; 
idade da criança na avaliação fonoaudiológica; idade no início da intervenção; se procurou outro serviço após o diagnóstico antes de ser atendido nesta instituição; quando iniciou e/ou terminou a escolarização; idade em que se adaptou AASI; se até o momento da coleta de dados continuava utilizando AASI.

A análise dos resultados desta pesquisa foi fundamentalmente quantitativa e a partir dos dados obtidos aspectos qualitativos foram discutidos. Na apresentação das tabelas foi realizada análise estatística descritiva dos dados, com cálculo da média aritmética, desvio-padrão e dos valores mínimo e máximo dos índices percentuais. Foi utilizado o software Statistical Package for Social Sciences (SPSS) $7.5^{(12)}$. Como os dados não apresentaram distribuição normal, foram utilizados testes não paramétricos (prova U de Mann Whitney).

\section{RESULTADOS}

Quanto a suspeita da perda auditiva, a idade variou de 2 meses a 14 anos de idade, sendo que em 34 casos $(10,6 \%)$ não houve suspeita da surdez. A média de idade foi de 1 ano e 9 meses (Figura 1).

A média de idade dos sujeitos para primeira consulta com o médico ou fonoaudiológico foi de 3 anos e 6 meses. O diagnóstico da surdez foi feito de 1 mês a 20 anos, sendo que média de idade foi de 4 anos e 3 meses. Os dois sujeitos diagnosticados com 1 mês de idade realizaram triagem auditiva neonatal. A idade para o início de intervenção variou entre 6 meses e 28 anos, com média de 6 anos e 1 mês.

Para a adaptação de AASI, a variação de idade foi de 11 meses a 42 anos, com média de 7 anos e 5 meses, conforme mostra a Figura 1. Os dados sobre idade mínima, máxima e a média de idade dos sujeitos desde o momento da suspeita até a adaptação de AASI podem ser vistos na Tabela 1.

Os dados sobre o período de suspeita, primeira consulta médica, diagnóstico, intervenção e adaptação de AASI serão apresentados separadamente para comparação entre os achados dos dois serviços selecionados para o estudo. A média de idade de suspeita da surdez foi superior nos usuários da clínica especializada do que aos usuários da clínica universitária (Tabela 2).

Em relação à idade do diagnóstico, observa-se que usuários da clínica especializada foram diagnosticados também
Tabela 1. Distribuição de dados referentes à idade dos sujeitos desde a suspeita da perda à adaptação do AASI

\begin{tabular}{lccccc}
\hline & $\mathrm{n}$ & Média & $\mathrm{DP}$ & Mínimo & Máximo \\
\hline Suspeita* $^{*}$ & 320 & 1,9879 & 2,1228 & 0,2 & 14,00 \\
Ida ao médico & 320 & 3,6371 & 3,3489 & 0,2 & 19,00 \\
Idade diagnóstica & 320 & 4,3305 & 3,5531 & $0,1^{* *}$ & 20,00 \\
Intervenção & 320 & 6,1795 & 4,3507 & 0,6 & 28,00 \\
Idade AASI & 320 & 7,5153 & 6,2845 & 0,11 & 42,00 \\
\hline
\end{tabular}

${ }^{*}$ Em 34 casos não houve suspeita; ${ }^{* *}$ crianças que fizeram TANU

Legenda: $\mathrm{n}=$ número amostral; $\mathrm{DP}=$ desvio-padrão

Tabela 2. Distribuição de dados referentes à idade dos sujeitos no momento da suspeita da surdez por Instituição

\begin{tabular}{lccccc}
\hline Instituição & $\mathrm{n}$ & Média & $\mathrm{DP}$ & Mínimo & Máximo \\
\hline Clínica universitária & 114 & 1,5610 & 1,7506 & 0,2 & 1,0 \\
Clínica especializada & 206 & 2,2242 & 2,2728 & 0,2 & 14,0 \\
\hline
\end{tabular}

Legenda: $\mathrm{n}=$ número amostral; $\mathrm{DP}=$ desvio-padrão

mais tardiamente quando comparados aos usuários da clínica universitária (Tabela 3).

Tabela 3. Distribuição de dados referentes à idade dos sujeitos no momento do diagnóstico de surdez por Instituição

\begin{tabular}{lccccc}
\hline Instituição & $\mathrm{n}$ & Média & $\mathrm{DP}$ & Mínimo & Máximo \\
\hline Clínica universitária & 114 & 3,8168 & 3,7223 & 0,1 & 18,0 \\
Clínica especializada & 206 & 4,6148 & 3,4321 & 0,5 & 20,00 \\
\hline
\end{tabular}

Legenda: $\mathrm{n}=$ número amostral; $\mathrm{DP}=$ desvio-padrão

Utilizando o teste U de Mann-Whitney, foi observado que há diferença entre a média de idade de diagnóstico dos usuários dos dois serviços (Figura 2).

A análise feita sobre a adaptação de AASI mostrou que a média de idade dos usuários da instituição especializada foi maior do que dos usuários da clínica universitária ( 8 anos e 2 meses e 7 anos, respectivamente). O teste de Mann-Whitney demonstra essa diferença $(\mathrm{p}<0,05)$ (Figura 3$)$.

\section{DISCUSSÃO}

A análise dos resultados mostrou que a idade média da

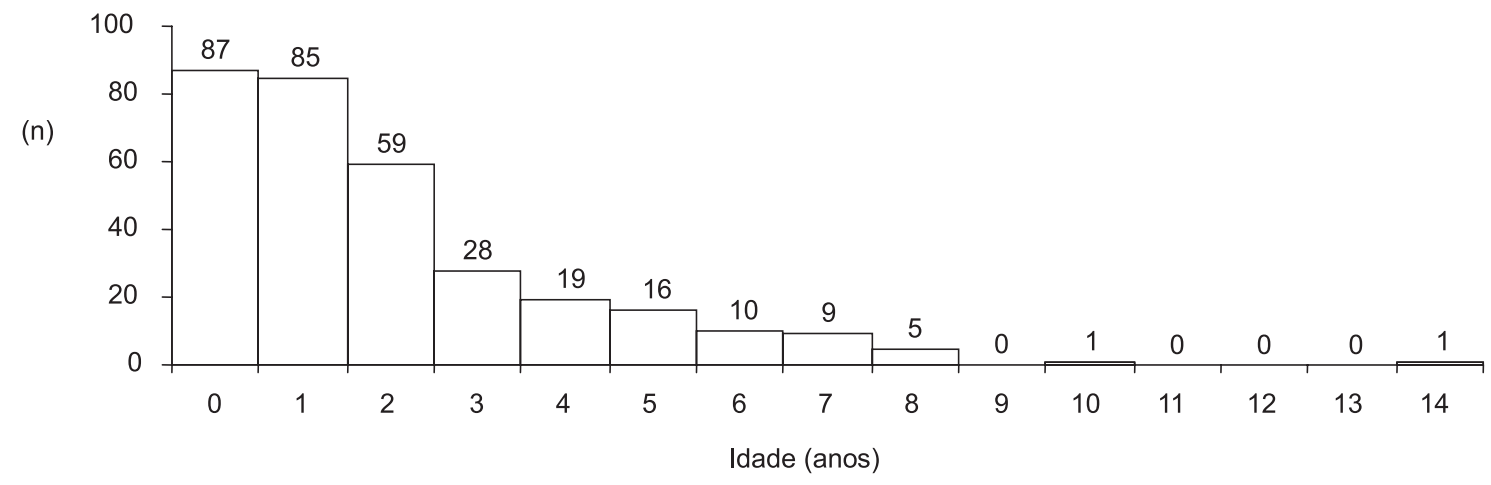

Legenda: $\mathrm{n}=$ número amostral

Figura 1. Distribuição dos casos quanto à idade da suspeita da perda auditiva 


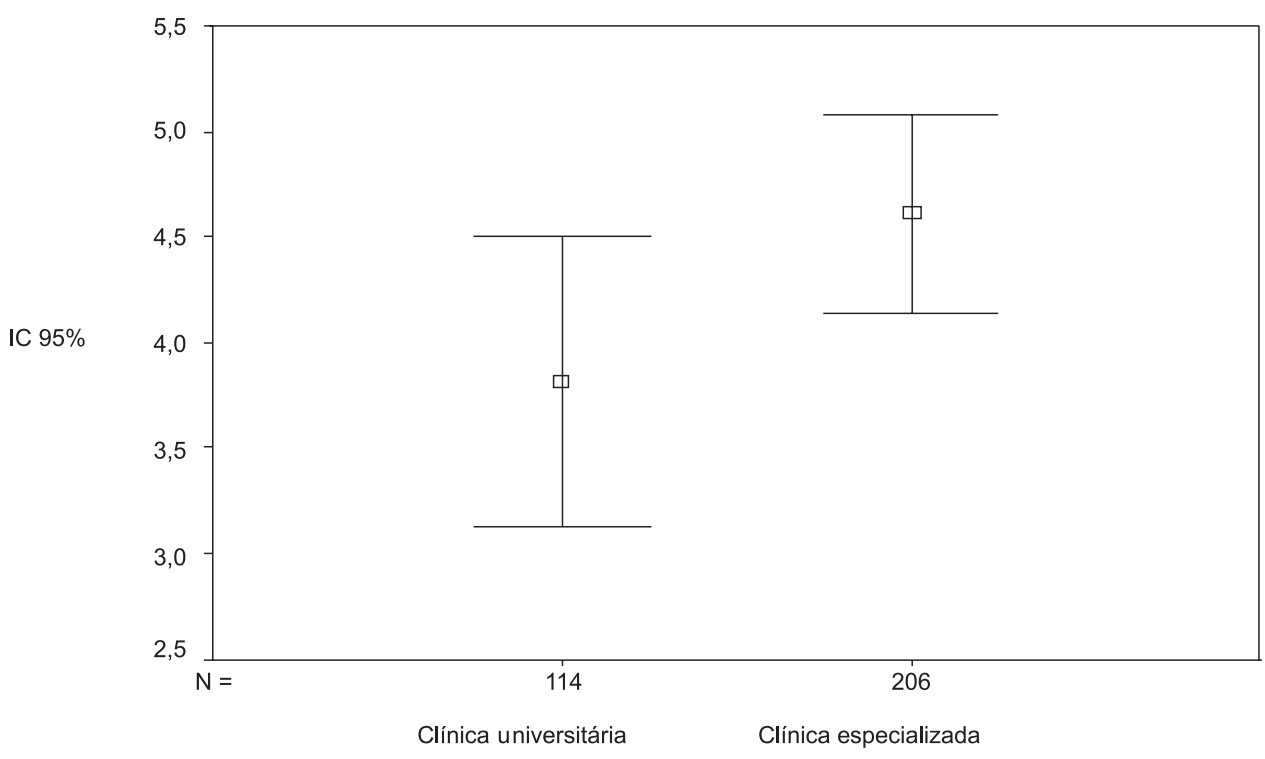

Legenda: $\mathrm{IC}=$ intervalo de confiança

Figura 2. Intervalo de confiança de $95 \%$ da idade (em anos) quando ocorreu o diagnóstico da surdez

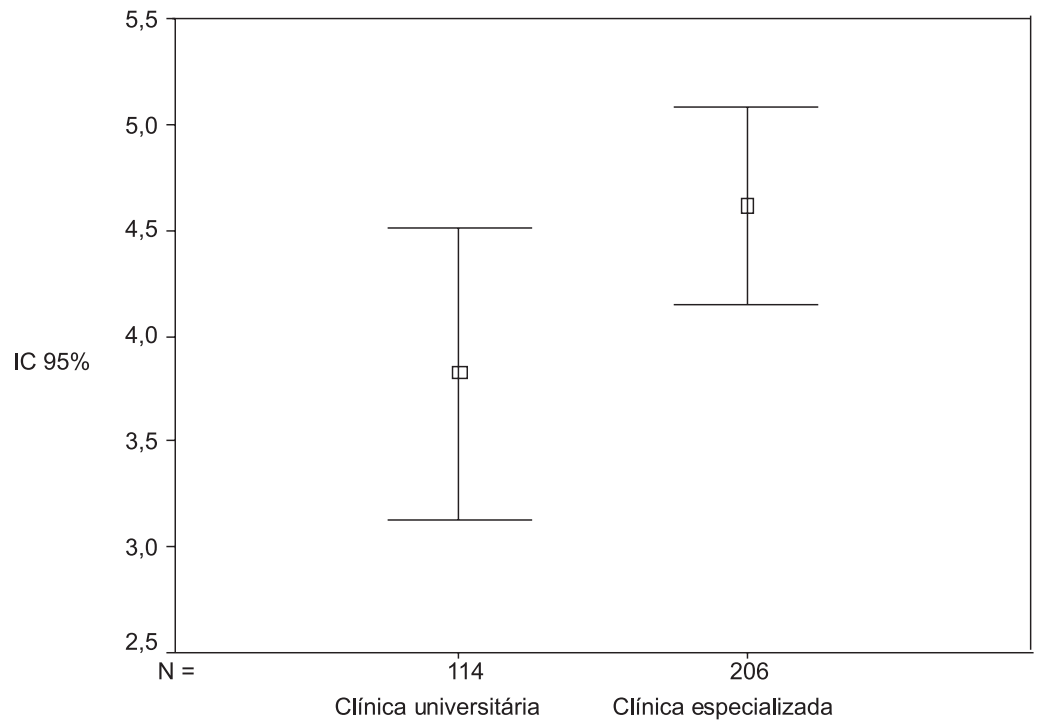

Legenda: $I C=$ intervalo de confiança

Figura 3. Intervalo de confiança de $95 \%$ da idade (anos), por instituição, quando iniciou o uso de AASI

suspeita, primeira consulta médica, diagnóstico, intervenção e adaptação de AASI ocorreram tardiamente em relação a outras pesquisas e aos padrões estabelecidos pelos comitês nacionais e internacionais, de que o diagnóstico aconteça entre o terceiro e o quarto mês de vida do bebê e a intervenção antes dos 6 meses. Cabe destacar que este estudo, quando comparado a outros já realizados, abrangeu uma população maior e mais heterogênea em relação à idade, e talvez tal fato tenha influenciado nos resultados obtidos ${ }^{(13,14)}$.

Verificou-se que a suspeita da surdez ocorreu em situação familiar na maioria dos casos com consequente mobilização para busca de diagnóstico, o que também foi constatado em outras pesquisas ${ }^{(15-19)}$. Este dado é alarmante, já que o número de suspeitas levantadas por profissionais da saúde foi muito baixo. Embora estas crianças frequentassem serviços de saúde pública como vacinação, puericultura e visitas aos pediatras, em poucos casos os profissionais se atentaram para as alterações auditivas, fato também observado em outros estudos ${ }^{(9,20,21)}$.

Observou-se que houve relação entre o período de suspeita e o tipo de perda auditiva. Perdas profundas foram suspeitadas com média de idade de 1 ano e 9 meses, perdas severas com 2 anos e 8 meses e perdas moderadas com 9 anos e 8 meses, dado também encontrado em outro estudo ${ }^{(18)}$.

Houve um grande intervalo de tempo entre a suspeita dos pais e o diagnóstico da surdez. Esse intervalo pode estar relacionado a uma desconfiança da família e ao mesmo tempo receio da confirmação dessa suspeita. Uma outra possibilidade é a dificuldade de acesso dos serviços de saúde que atendem essa 
demanda, com filas de espera para o atendimento otorrinolaringológico e avaliação audiológica, bastante comum na região.

A idade média da confirmação do diagnóstico da surdez ocorre por volta de 4 anos e 3 meses, trazendo implicações importantes para a aquisição da linguagem. Durante o desenvolvimento do sistema nervoso, todos os sistemas sensoriais, especialmente as vias nervosas, maturam ao mesmo tempo em que o sistema motor e os processos mentais. Desse modo, se existe uma deficiência auditiva não atendida na fase de maturação (compreendida entre 0 e 3 anos), ocorrerão alterações que dificilmente serão corrigidas mais tarde, podendo gerar comprometimento no desenvolvimento das crianças nas esferas escolar, social e familiar ${ }^{(22)}$. Trata-se de um diagnóstico bastante tardio da população do presente estudo, revelando uma realidade que merece maior atenção daqueles interessados em saúde e qualidade de vida.

Os dados mostram diferença entre a idade média de diagnóstico dos usuários dos dois serviços; usuários da clínica especializada foram diagnosticados mais tardiamente quando comparados aos usuários da clínica universitária. Este dado não era esperado, pois a clínica universitária não é um centro de diagnóstico, mas de atendimento, já que não dispõe de todos os equipamentos para exames objetivos, dentro outros aspectos. Assim, quando há necessidade de exames específicos para fechar um diagnóstico audiológico, são feitos encaminhamentos aos equipamentos especializados da região, que frequentemente requerem certo tempo de espera em filas para a realização dos exames. É válido ressaltar que a clínica especializada é um dos equipamentos aos quais os usuários da clínica universitária se remetem para o diagnóstico audiológico. Deste modo, seria esperado que este centro fosse mais ágil na detecção e realização de diagnósticos mais precoces, o que não condiz com os resultados obtidos.

Outro ponto a destacar é que o município de Campinas, onde se localiza um dos serviços pesquisados, é de grande porte, concentra um grande número de indústrias de ponta e exibe feições de metrópole. É considerada, de acordo com a Secretaria de Economia e Planejamento do Governo do Estado de São Paulo, uma região administrativa que possui grande oferta de serviços de saúde e a maior parte das demandas por serviços de qualquer natureza pode ser, satisfatoriamente, coberta sem precisar sair do município ${ }^{(23)}$.

No município de Campinas, foi regulamentada a Lei n ${ }^{\circ} .10759$ em 16/02/2004 que obriga todas as maternidades e estabelecimentos hospitalares congêneres do município a realizar exames de detecção de surdez em recém-nascidos no período neonatal ou em até 60 dias a contar do nascimento. Segundo a Portaria $n^{\circ} 432$ 14/11/2000, os procedimentos realizados na Triagem Auditiva Neonatal Universal (TANU) são de alta complexidade e devem ser cobrados através de Autorização para Procedimentos de Alta Complexidade (APAC), mas apesar disso o serviço não é oferecido de forma universal aos usuários em muitas maternidades.

A idade média de adaptação do AASI encontrada também pode ser considerada tardia. Tal resultado serve como alerta, pois essa etapa ainda está bem longe da idade considerada ideal, que é até os seis meses de vida ${ }^{(3,4,9,21)}$. A adaptação do AASI deve ser realizada assim que se conclui o diagnóstico audio- lógico ${ }^{(24-25)}$, como recurso para evitar mais tempo de privação sensorial e iniciar a intervenção fonoaudiológica.

\section{CONCLUSÃO}

Ao término deste estudo, a apreciação crítica dos resultados permitiu concluir que a suspeita, primeira consulta médica, diagnóstico, intervenção e adaptação de AASI ocorrem tardiamente, se considerarmos os padrões de indicação preconizados na atualidade. Há um tempo longo de espera para início de fonoterapia, indicando que os recursos públicos oferecidos são insuficientes e consequentemente lentos, o que interfere no processo de reabilitação da criança surda.

Apesar da aprovação de projetos de leis e triagem auditiva, os programas de identificação precoce da perda auditiva não têm atingido a população como um todo, bem como o diagnóstico, intervenção e adaptação de AASI antes do sexto mês de vida. A diferença estatisticamente significante encontrada entre os dois equipamentos, em relação às médias de idade do diagnóstico da surdez e adaptação de AASI revela-se como um dado preocupante, instituições consideradas como referência no atendimento em surdez não têm conseguido realizar os procedimentos necessários em períodos adequados, sugerindo que uma investigação mais detalhada dos problemas enfrentados seria importante para alcançar um padrão mais adequado de atendimento.

A Política Nacional de Atenção à Saúde Auditiva foi iniciada de forma mais estruturada na região de Campinas em 2004, e isto deve colaborar para alterar esta realidade. Todavia, os dados coletados não mostraram o impacto desta medida que ainda é bastante recente.

\section{REFERÊNCIAS}

1. Gomes-Machado ML, Soares AD, Chiari BM. Avaliação dinâmica e interdisciplinar na deficiência auditiva em ambulatório público: relato de caso. Rev Soc Bras Fonoaudiol. 2009;14(3):416-20.

2. Pádua FGM, Marone S, Bento RF, Carvallo RMM, Durante AS, Soares JC, et al. Triagem auditiva neonatal: um desafio para sua implantação. Arq Otorrinolaringol. 2005;9(3):190-4.

3. Yoshinaga-Itano C, Apuzzo ML. Identification of hearing loss after age 18 months is not early enough. Am Ann Deaf. 1998;143(5):380-7.

4. Yoshinaga-Itano C, Sedey AL, Coulter DK, Mehl AL. Language of early- and later-identified children with hearing loss. Pediatrics. 1998;102(5):1161-71.

5. Downs MP, Yoshinaga-Itano C. The efficacy of early identification and intervention for children with hearing impairment. Pediatr Clin North Am. 1999;46(1):79-87.

6. Vieira ABC, Macedo LR. O diagnóstico da perda auditiva na infância. Pediatria (São Paulo). 2007;29(1):43-9.

7. Yoshinaga-Itano C, Sedey A. Language, speech and social-emotional development of children who are deaf or hard of hearing: the early years. Volta Rev. 2000;100(5):213-34.

8. Ferro L, Gonçalves I, Cieri CC. Tempo de latência entre suspeita, diagnóstico e intervenção em crianças portadoras de deficiência auditiva na cidade de Campo Grande - MS. J Bras Fonoaudiol. 2002;3(11):10813.

9. Tschiedel RS, Bandini HHM, Bevilacqua MC. Diagnóstico da deficiência auditiva na infância: uma avaliação do nível de conhecimento dos pediatras de uma cidade da região Centro-Oeste paulista. Pediatr Mod. 2000;36(9):607:610:614:617:passim-607-612-615-618. 
10. Lima MCMP, Rossi TRF, Françozo MFC, Marba ST, Lima GML, Santos MFC. Detecção de perdas auditivas em neonatos de um hospital público. Rev Soc Bras Fonoaudiol. 2010;15(1):1-6.

11. Regiões Administrativas e Metropolitanas do Estado de São Paulo. [Internet] [citado 2006 Nov 2]. Disponível: http//:www.igc.sp.gov.br/mapasRas.htm

12. Bisquerra R, Sarriera JC, Martínez F. Introdução à estatística: enfoque informático com o pacote estatístico SPSS. Porto Alegre: Artmed; 2004. 255p.

13. Barreira-Nielsen C, Futuro Neto HA, Gattaz G. Processo de implantação de Programa de Saúde Auditiva em duas maternidades públicas. Rev Soc Bras Fonoaudiol. 2007;12(2):99-105.

14. Novaes LHVS, Martinez ZO, Diniz E, Xaubet RS, Carteri G, Munhoz C, Zanotelli V. Avaliação e monitoração das respostas auditivas do neonato a sons calibrados. Rev Bras Otorrinolaringol. 1997;63(2):122-30.

15. Remencius NR. Aspectos de acessibilidade de serviços públicos de saúde no atendimento a crianças portadoras de deficiência auditiva [dissertação]. São Paulo: Pontifícia Universidade Católica de São Paulo; 1998.

16. Françoso MFC, Barbalho RS. Abandono do atendimento institucional por parte das famílias de crianças surdas. In: Anais do VII Encontro Nacional de Pesquisadores em Serviço Social; 200021 - 24 novembro; Brasília, Brasil. Brasília: Universidade de Brasília; 2000. p. 506-14.

17. Meyer ASA. Caracterização dos aspectos diagnósticos e de intervenção das crianças atendidas nos serviços de deficiência auditiva do HRAC/ USP-Bauru [tese]. Bauru: Universidade de São Paulo; 2003.

18. Heck F, Raymann BCW. Tempo decorrido entre a suspeita da surdez, a primeira ida ao médico e/ou fonoaudiólogo, o diagnóstico e o início da reabilitação com crianças surdas. J Bras Fonoaudiol. 2003;4(16)175-85.

19. Helfer TM, Lee RB, Maris DC, Shields AR. Wanted: a national standard for early hearing detection and intervation outcomes data. Am J Audiol. 2003;12(1):23-30.

20. Linares AE, Feniman MR. O que pediatras e obstetras gostariam de saber sobre a prevenção da deficiência auditiva na infância. Pediatr Mod. 2003;39(6)187-92.

21. Olusanya BO, Luxon LM, Wirz SL. Benefits and challenges of newborn hearing screening for developing countries. Int J Pediatr Otorhinolaryngol. 2004;68(3):287-305. Erratum in: Int J Pediatr Otorhinolaryngol. 2004;68(4):517.

22. Governo do Estado de São Paulo. Secretaria da Economia e Planejamento. [Internet]; c2009. Região Metropolitana de Campinas. [citado 2009 Dez 11]. Disponível em: http://www.planejamento.sp.gov. $\mathrm{br} / \mathrm{des} / \mathrm{textos} 8 / \mathrm{RMC}$.

23. Comitê Brasileiro sobre Perdas Auditivas na Infância. Recomendação 01/99 do Comitê Brasileiro sobre perdas auditivas na infância. J Cons Fed Fonoaudiol. 2000;(5):3-7.

24. Delb W, Merkel D, Pilorrget K, Schmitt J, Plinkert PK. Effectiveness of a TEOAE- based screening program. Can a patient-tracking system effectively be organized using modern information technology and central data management? Eur Arch Otorhinolaryngol. 2004;261(4):191-6.

25. Finitzo T, Grosse $S$. Quality monitoring for early hearing detection and intervention programs to optimize performance. Ment Retard Dev Disabil Res Rev. 2003;9(2):73-8.

\section{Anexo 1. Protocolo para coleta de dados}

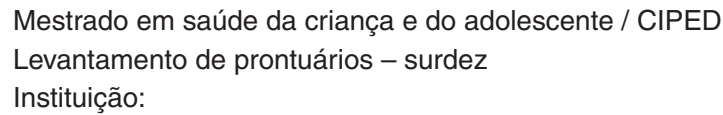

Data:

Nome:

Data de nascimento:

Profissão da mãe:

Profissão do pai:

Cidade:

Quando foi a suspeita da surdez (idade da criança):

Como foi a suspeita da surdez:
( ) Arquivo morto

Idade:

Escolaridade:

Escolaridade da mãe:

Escolaridade do pai:

\section{Quem suspeitou:}

Idade da criança na $1^{\text {a }}$ ida ao médico / fgo:

\section{Especialidade:}

Diagnóstico: OD / OE / AO ( ) sensorioneural ( ) condutiva ( ) mista ( ) normal

OD: ( ) leve ( ) moderada ( ) severa ( ) profunda ( ) anacusia

OE: ( ) leve ( ) moderada ( ) severa ( ) profunda ( ) anacusia

Etiologia da surdez:

Data da avaliação fonoaudiológica:

Data do início da intervenção:

Procurou outro serviço após o diagnóstico?:

Data do início e/ou término de escolarização:

Data do desligamento da instituição e motivo:

Uso de prótese (s):

( ) $\operatorname{Sim}$ ( ) Não Desde quando?

( ) OD ( ) OE ( ) AO

Ainda utiliza próteses (s)?: ( ) Sim （ ) Não Último dado:

Tempo decorrido entre: $\quad$ Suspeita da surdez e $1^{\mathrm{a}}$ ida ao médico:

$1^{\mathrm{a}}$ ida ao médico e diagnóstico:

Diagnóstico e início de intervenção:

Observações: 